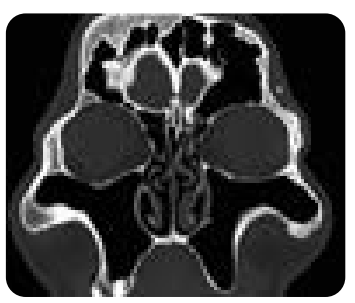

\title{
HALLAZGOS \\ RADIOLÓGICOS \\ CRANEOFACIALES EN \\ ACROMEGALIA
}

Craniofacial Radiological Findings In Acromegaly

\section{$\boldsymbol{\nu}$}

\section{Palabras clave (DeCS)}

Acromegalia

Hormona del crecimiento

Imagen por resonancia

magnética

\section{Key words (MeSH)}

Acromegaly

Growth hormone

Magnetic resonance

imaging
Médico residente de radiología e imágenes diagnósticas. Fundación Universitaria Sanitas. Departamento de Radiología e Imágenes Diagnósticas. Clínica Universitaria Colombia, Clínica Reina Sofía. Bogotá, Colombia.

${ }^{2}$ Neurorradióloga. Departamento de Radiología e Imágenes Diagnósticas, Clínica Reina Sofía. Docente adscrita de la Fundación Universitaria Sanitas. Bogotá, Colombia.

Luis Manuel Alejandro Acosta Rosas Catalina Wilches Vanegas²

\section{Resumen}

La primera descripción médica de la acromegalia se hizo en 1886, por el neurólogo francés Pierre Marie. Esta enfermedad, poco frecuente, generalmente la causa un adenoma hipofisario, que activa un fenotipo característico de sobrecrecimiento, causa desproporciones craneofaciales y complicaciones sistémicas en especial en los sistemas cardiovascular, respiratorio y metabólico, secundario a los niveles excesivos de hormona del crecimiento y factor de crecimiento similar a la insulina. La detección radiológica de estos hallazgos craneofaciales, mediante estudios por tomografía y resonancia magnética, son de gran importancia, puesto que brindan a estos pacientes un diagnóstico precoz de la enfermedad, lo cual conlleva instaurar un tratamiento oportuno de esta patología.

\section{Summary}

The first medical description of acromegaly was in 1886, by French neurologist Pierre Marie. This rare disease is usually caused by a pituitary adenoma, which has a typical phenotype of overgrowth, craniofacial disproportions and systemic complications, specially in the cardiovascular, respiratory and metabolic systems, secondary to excessive levels of growth hormone and insulin-like growth factor. Radiological detection (computed tomography and magnetic resonance imaging) of these craniofacial features is important, as they provide patients with an early diagnosis and treatment of disease.

\section{Introducción}

En 1886, el neurólogo francés Pierre Marie hizo la primera descripción médica de la acromegalia. Él acuñó el término acromegalia, que proviene del francés y significa hipertrofia de las extremidades, pues esta entidad se caracteriza por la hipertrofia de manos, pies y cara. Posteriormente, se descubrió que prácticamente todos los pacientes con acromegalia tenían aumento de tamaño en la hipófisis, y que la hiperfunción de la misma era secundaria a un tumor pituitario (1). Años después se descubrió que la causa de la acromegalia era aumento en la hormona del crecimiento $(\mathrm{GH}) \mathrm{y}$ del factor de crecimiento similar a la insulina (IGF-I). A principios del siglo XX se realizaba resección quirúrgica de la hipófisis y radioterapia como tratamientos para los pacientes con acromegalia. Después de 1970 se introdujo la terapia médica con agonistas dopaminérgicos en primer lugar, seguidos por los análogos de la somatostatina y los bloqueadores de los receptores de GH (1).

Es una enfermedad poco frecuente, que suele ser subdiagnosticada (2). La causa es un adenoma de la glándula pituitaria, en más del $95 \%$ de los pacientes y en el $5 \%$ restante, es secundaria a un tumor hipotalámi- 
co o neuroendocrino (generalmente de origen pulmonar o pancreático) lo que conduce a hiperplasia somatotrófica y acromegalia $(2,3)$. Produce deformidades de manera progresiva y ocasiona una amplia gama de complicaciones sistémicas, algunas de las cuales son responsables del aumento de la mortalidad de los pacientes no tratados (4).

La gran mayoría de los adenomas pituitarios secretores de $\mathrm{GH}$ son esporádicos. Sin embargo, la acromegalia también puede tener un patrón hereditario, por asociación con otras anomalías endocrinas (síndromes de neoplasias endocrinas múltiples tipo 1 y tipo 4) o aparecer como un adenoma hipofisario familiar aislado FIPA (familial isolated pituitary adenoma, por sus siglas en ingles), que se debe a mutaciones en la línea germinal del gen AIP (1). Otra entidad relacionada es el acrogigantismo ligado a X (XLAG), causado por microduplicaciones en el cromosoma Xq26.3, que abarca el gen GPR101, el cual está altamente regulado en los tumores pituitarios (2). La acromegalia también afecta alrededor del $20 \%$ de los pacientes con síndrome de McCune-Albright (2).

En los pacientes con acromegalia se manifiesta un fenotipo característico de sobrecrecimiento y desproporciones craneofaciales. Esto se debe a que los altos niveles del factor de crecimiento similar a la insulina ocasionan un exceso en el crecimiento de diversos tejidos epiteliales y conectivos, vísceras, el sistema cardiovascular, pulmonar y la piel; adicionalmente, genera neoformación de periostio, lo cual resulta en la hipertrofia característica del hueso nasal, el crecimiento excesivo de la mandíbula, el ensanchamiento maxilar y la prominencia del hueso frontal. Se ha informado disminución en la distancia intercarotídea (distancia entre las arterias carótidas comunes derecha e izquierda). Adicionalmente, el exceso de GH y de IGF-1 conllevan otras patologías, como pólipos en el colon, problemas cardiovasculares, apnea, visceromegalia, alteraciones metabólicas $y$ endocrinas $(5,6)$.

\section{Epidemiología}

Los datos epidemiológicos descritos en la literatura estiman una prevalencia de 60 casos por millón y una incidencia anual entre 3 a 4 pacientes por millón $(5,6)$. Otros estudios informan una prevalencia total que oscila entre 2,8 y 13,7 casos por 100.000 habitantes con una incidencia entre 0,2 y 1,1 casos/ 100.000 habitantes (4). Se ha establecido que las tasas más elevadas encontradas en los estudios recientes pueden atribuirse a la mayor conciencia de la enfermedad y a los avances en las herramientas de diagnóstico $(4,7)$. Afecta por igual a hombres y mujeres $(5,6)$.

La edad media al momento del diagnóstico es la quinta década de la vida, la cual oscila entre 40,5 y 47 años (hombres: 36,5-48,5 años y mujeres: 38-56 años), los pacientes menores de 20 años representan hasta el $5 \%$ de los casos $(2,4)$.

Los pacientes con acromegalia presentan una tasa de mortalidad aumentada del $30 \%$, secundaria a enfermedades cardiovasculares que representan la causa de muerte en el $60 \%$ de los casos, seguida por enfermedades respiratorias en el $25 \%$ y neoplasias en el $15 \%$ de ellos $(4,8)$.

\section{Manifestaciones clínicas}

Las manifestaciones clínicas más frecuentes son el sobrecrecimiento distal de las extremidades $(78,8-85,7 \%)$ y rasgos faciales toscos
(71,2-71,4\%), macroglosia, engrosamiento cutáneo con síntomas como cefalea, hiperhidrosis, artralgias, ronquidos, astenia y síndrome del túnel del carpo (7).

Las manifestaciones clínicas en los pacientes con acromegalia se presentan por dos mecanismos: por efecto local de una masa pituitaria en expansión y por los efectos directos e indirectos de la secreción excesiva de GH e IGF-1, que conducen a complicaciones sistémicas y deterioro, de la calidad de vida(2).

La cefalea, uno de los efectos locales del tumor, se encuentra en aproximadamente el $60 \%$ de los pacientes; tiende a ser intensa y desproporcionada respecto al tamaño del tumor, puede estar relacionada con el estiramiento de la duramadre secundario al crecimiento tumoral, con la invasión del seno cavernoso e irritación del nervio trigémino o con la hipersecreción de la $\mathrm{GH}(2,8)$.

En los casos en los que los adenomas hipofisarios comprimen el quiasma óptico se generaran alteraciones del campo visual, las cuales comienzan en la mitad superior de la periferia de los campos visuales temporales superiores, y luego progresan a hemianopsia bitemporal. La compresión persistente puede resultar en ceguera (2).

Otras características neurológicas, como hidrocefalia, exoftalmos unilateral (debido a la invasión orbital) y convulsiones, se observan en los casos de adenomas hipofisarios de gran tamaño (2).

Dentro de las manifestaciones sistémicas se encuentran:

Crecimiento acral: En los pacientes con acromegalia se evidencia un crecimiento excesivo en las manos y los pies, casi de forma patognomónica; esto se da por un edema en los tejidos blandos. La osteoartritis y la hiperlaxitud articular también pueden contribuir a las deformidades de las manos (2).

Cambios en la piel y apéndices: Estas alteraciones contribuyen a las deformidades faciales y acrales. El engrosamiento de la piel es prominente en la cara, las manos y los pies, y se atribuye a los depósitos de glucosaminoglucanos, aumento de la producción de colágeno del tejido conjuntivo y edema $(2,4)$.

El aumento de tamaño de las glándulas sebáceas conduce a hiperhidrosis y bromhidrosis, los cuales pueden ser signos tempranos de la enfermedad y se presentan en un $70 \%$ de los casos, también se puede observar acantosis nigricans por resistencia a la insulina, psoriasis, fenómeno de Raynaud, hirsutismo e hipertricosis (2).

Cambios musculoesqueléticos: La artropatía acromegálica puede afectar hasta el $84 \%$ de los pacientes, con inflamación de las articulaciones, hiperlaxitud y engrosamiento cartilaginoso. Hay un mayor riesgo de fracturas por compresión vertebral que pueden acelerarse por el hipogonadismo (2). Adicionalmente, neuropatía periférica con alteraciones sensoriales en las manos y los pies, como el síndrome del túnel del carpo, entre el 20-64\% de los pacientes (2). Cambios osteoartrósicos con remodelación ósea, formación de osteofitos, quistes subcondrales, disminución de los espacios articulares y laxitud del ligamento periarticular (2). El dolor musculoesquelético es frecuente y afecta la calidad de vida de estos pacientes (2). 

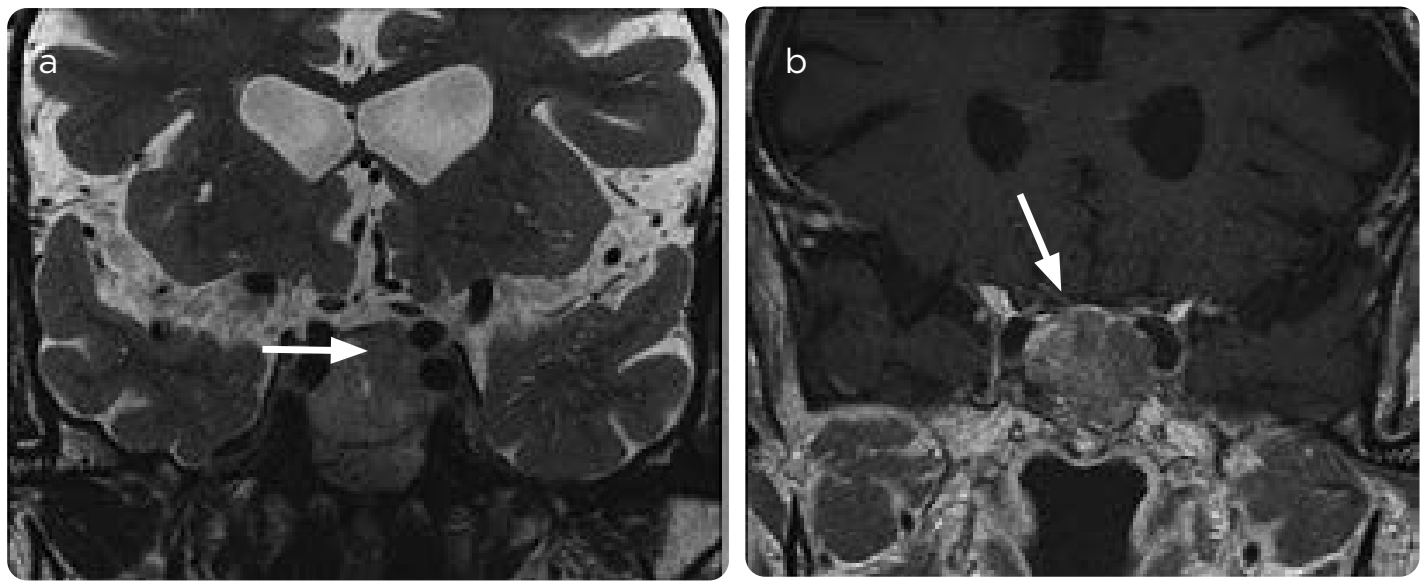

Figura 1. a) RM, coronal con información T2: se observa lesión expansiva heterogénea que ocupa y expande la silla turca y se extiende en sentido lateral contactando ambos senos cavernosos: corresponde a un macroadenoma hipofisario. b) RM, coronal con información T1 con medio de contraste: la lesión realza el medio de contraste de forma heterogénea (flechas).

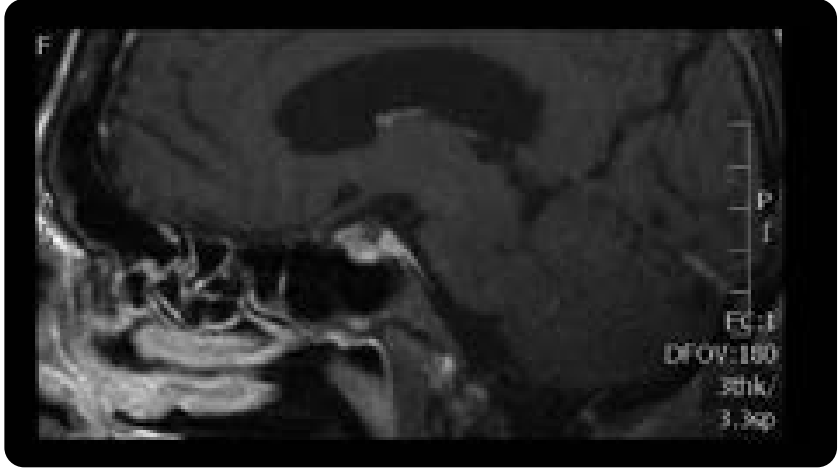

Figura 2. RM, sagital con información T1 con medio de contraste: se identifica alteración en la morfología de la silla turca, ausencia de adenohipófisis e interrupción del infundíbulo en un paciente con acromegalia.

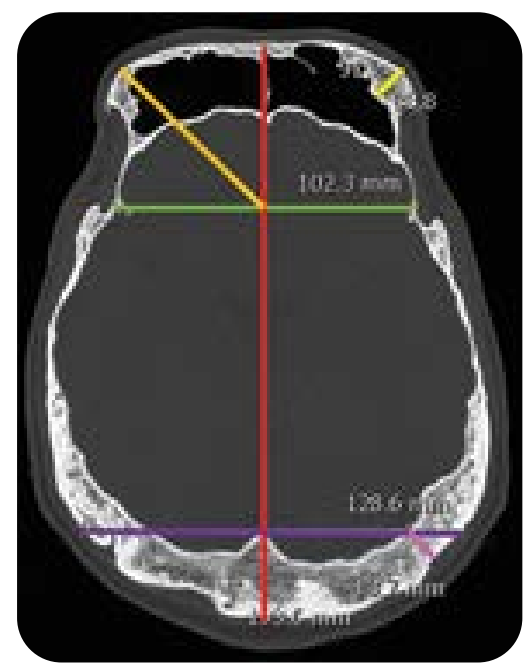

Figura 3. TAC en ventana de hueso: se muestra la forma adecuada para la medición del espesor de la tabla ósea frontal y occipital. Al nivel más alto de las alas mayores del esfenoides se traza una línea que conecta estos dos reparos anatómicos (línea verde) y una línea perpendicular a esta (línea roja), se traza una bisectriz desde la intersección de estas líneas hacia el hueso frontal (línea naranja) y en este punto se realiza la medición del espesor de la bóveda craneal frontal (línea amarilla). Para realizar la medición del espesor occipital, se traza una línea transversal a la altura de la proyección ósea más anterior del hueso occipital (línea morada), posteriormente trazar una línea en ángulo recto equidistante entre la cortical interna y la cortical externa de la bóveda craneal occipital (línea fucsia).
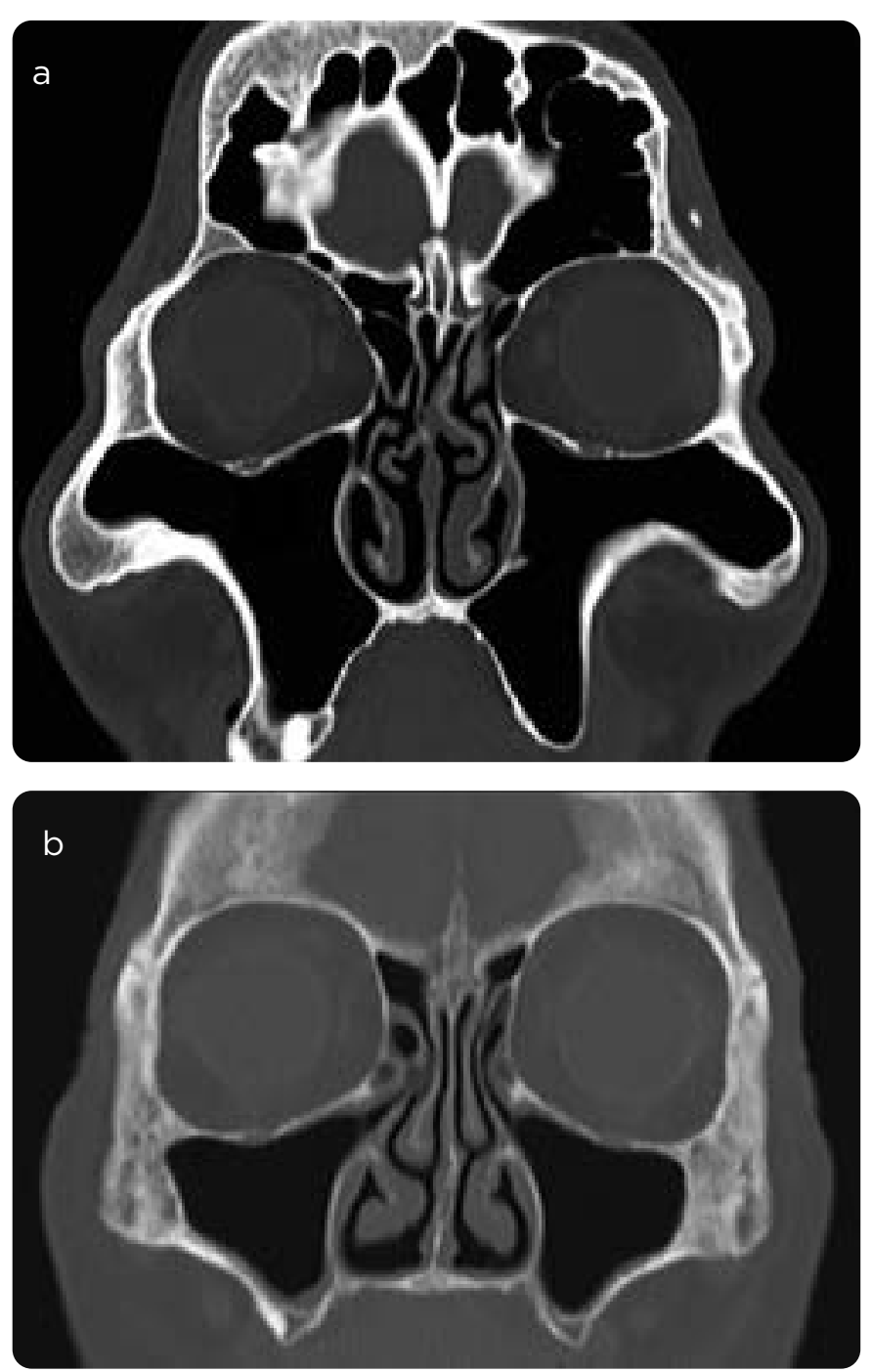

Figura 4. a) Reconstrucción coronal de escanografía en ventana de hueso: aumento del tamaño de los senos maxilares y frontales por hiperneumatización en un paciente con acromegalia. b) Reconstrucción coronal de escanografía en ventana de hueso, en un paciente sin acromegalia: nótese el tamaño normal de los senos maxilares en comparación con la imagen a). 

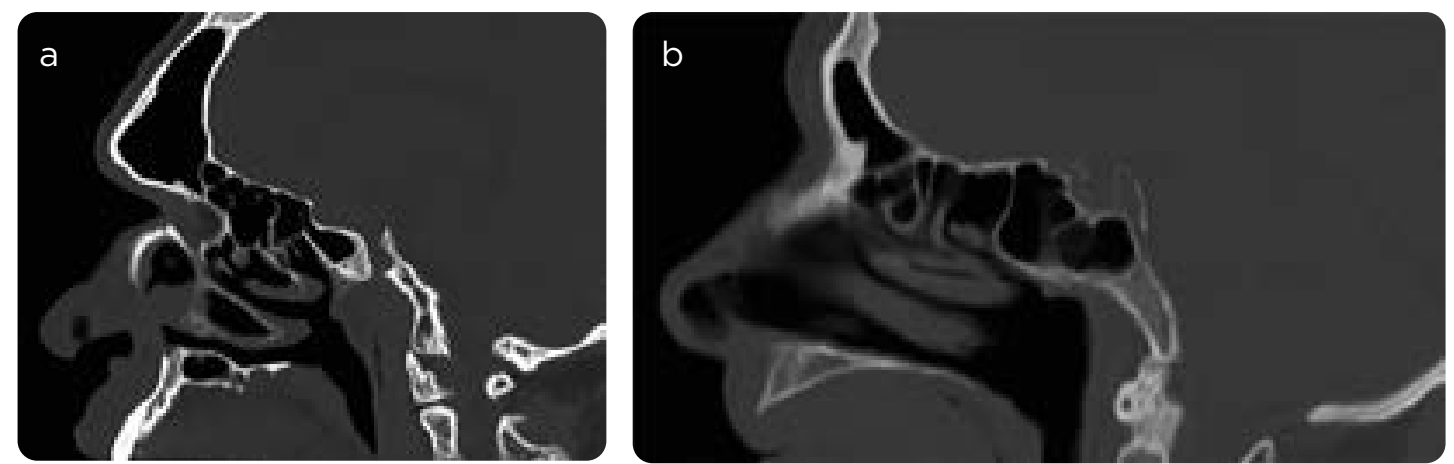

Figura 5. a) Reconstrucción sagital de escanografía en ventana de hueso: se observa el aumento del diámetro del seno frontal por hiperneumatización en un paciente con acromegalia. b) Reconstrucción sagital de escanografía en ventana de hueso, en un paciente sin acromegalia: nótese la diferencia en el tamaño del seno frontal en comparación con la imagen a).

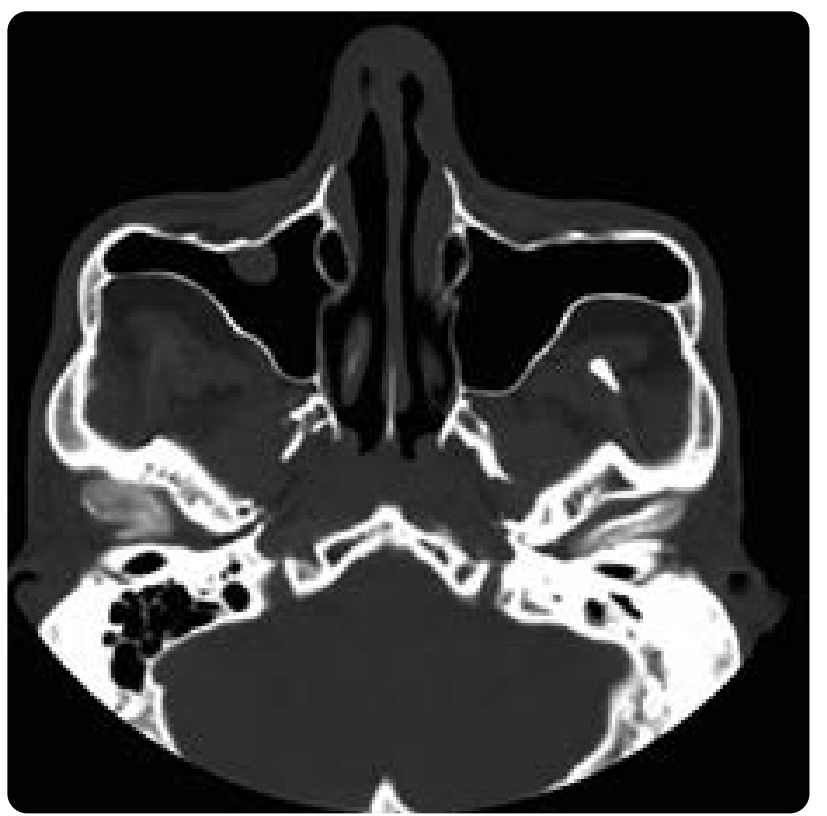

Figura 6. TAC en ventana de hueso de un paciente con acromegalia: se observa un aumento del diámetro de los senos maxilares y, adicionalmente, en el derecho, un pólipo. Estos son hallazgos frecuentes en los pacientes con acromegalia asociados a la hiperneumatización de los senos paranasales.

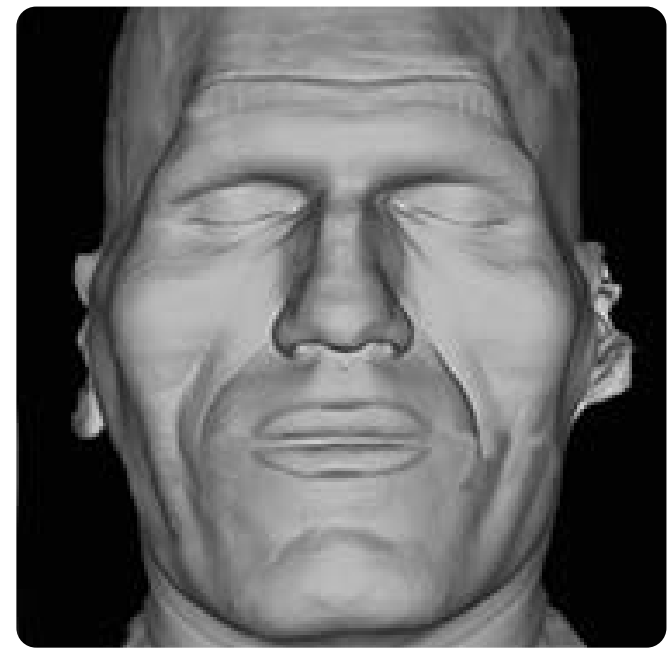

Figura 7. Reconstrucción 3D de escanografía de cara de un paciente con acromegalia con algunas malformaciones faciales típicas, como prognatismo, ensanchamiento del maxilar, prominencia superciliar e hipertrofia nasal.
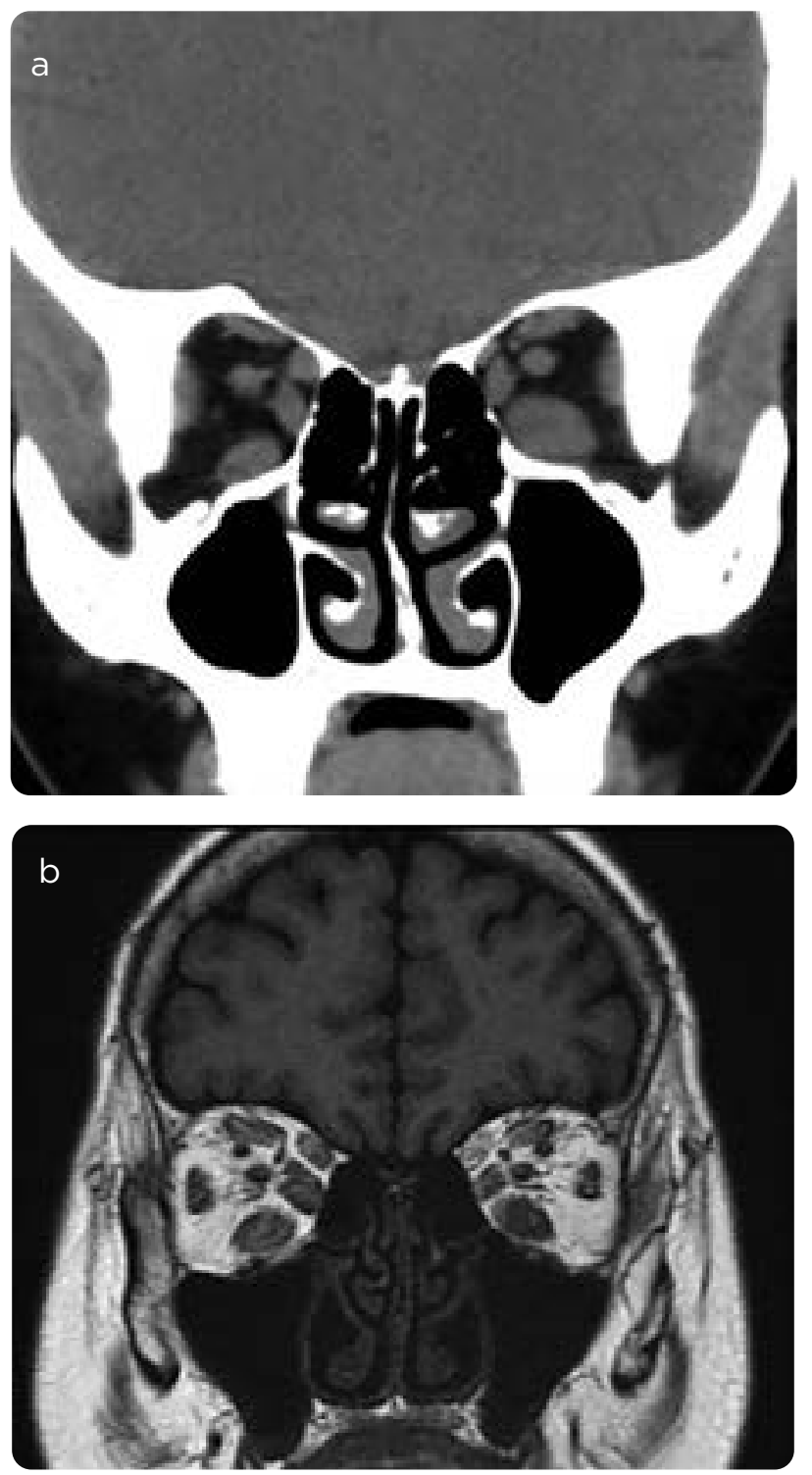

Figura 8. a) Reconstrucción coronal de escanografía de un paciente con acromegalia: engrosamiento difuso y bilateral de los músculos orbitarios, en especial de los rectos inferiores. b) RM, coronal con información T1 simple de un paciente con acromegalia: engrosamiento difuso y bilateral de los músculos extraoculares. 

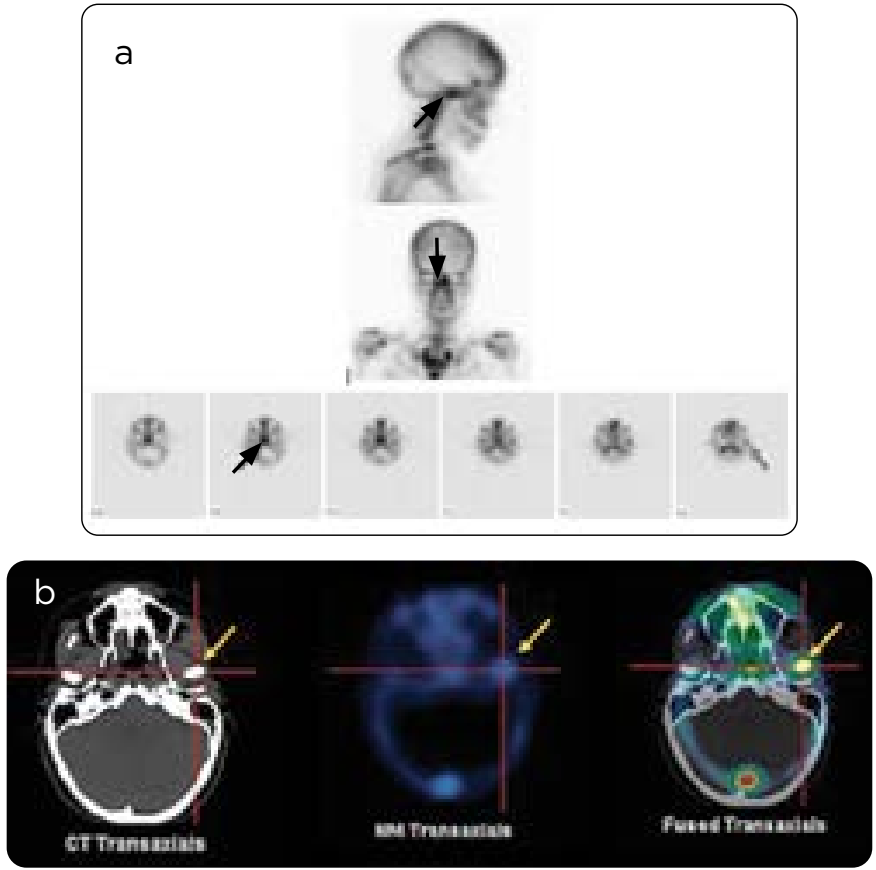

Figura 9. a) Gammagrafía ósea con SPECT: se observa hipercaptación de la silla turca (flecha roja) y del cóndilo mandibular izquierdo (flecha verde) en un paciente con acromegalia con un adenoma hipofisario e hiperplasia condílea. b) Gammagrafía ósea fusión SPECT CT del mismo paciente: se confirma la hiperplasia condílea activa izquierda (flecha amarilla).

\section{Complicaciones de la acromegalia}

- Cardiovasculares: La característica más común de la cardiomiopatía acromegálica es la hipertrofia biventricular concéntrica, ocurre principalmente en ausencia de hipertensión, pero puede agravarse por hipertensión y por las anomalías del metabolismo de la glucosa. En estadios posteriores se puede encontrar disfunción sistólica y diastólica de esfuerzo, así como anomalías valvulares $(2,4)$.

Aproximadamente, el $40 \%$ de los pacientes acromegálicos sufren arritmias como fibrilación auricular paroxística, taquicardia supraventricular paroxística y bloqueos de rama(4). Estos pacientes tienen varios factores de riesgo para isquemia miocárdica, tales como: niveles elevados de lipoproteína-a, triglicéridos, fibrinógeno, inhibidor del activador del plasminógeno y activador del plasminógeno tisular; adicionalmente, la hipertensión arterial se informar en el 30-40\% de los pacientes, probablemente debido al aumento del volumen plasmático, a la disminución de los niveles de péptido natriurético auricular (PNA), a la resistencia a la insulina y a la diabetes mellitus (4).

- Respiratorias: Entre el 20 y el $80 \%$ de los pacientes acromegálicos pueden manifestar apnea del sueño, con ronquidos, sueño fragmentado, somnolencia diurna y cefalea matutina(4). La apnea del sueño puede predisponer a cardiopatía isquémica, arritmias, hipertensión arterial y accidentes cerebrovasculares. El tipo obstructivo se ve en dos tercios de los pacientes afectados (debido al aumento del tejido blando en la nasofaringe y otras estructuras de las vías respiratorias superiores); en el tercio restante se da de tipo central (probablemente debido a los efectos directos en el centro de respiración de los elevados niveles de GH y de IGF-1) (4).
El estrechamiento de las vías respiratorias, el trastorno de los músculos respiratorios, la escoliosis en la columna torácica, el crecimiento excesivo de los pulmones y el aumento del volumen pulmonar, generan disfunción respiratoria, que lleva generalmente a enfisema y bronquiectasia (4).

- Metabólicas: El exceso de GH se asocia con la resistencia a la insulina, aumento de gluconeogénesis, lipólisis y disminución de la captación periférica de glucosa $(2,4)$, lo cual genera, a menudo, intolerancia a la glucosa, diabetes mellitus (hasta $28-46 \%$ de los casos) y dislipidemia (particularmente hipertrigliceridemia) y una elevación del LDL (2).

- Neoplasias: En los hombres con acromegalia se aumenta el riesgo de desarrollar adenomas de colon después de los 50 años de edad (4). El riesgo de enfermedad nodular tiroidea y cáncer de tiroides se incrementa en la acromegalia con un OR de hasta 7,5 (2). La acromegalia predispone a la hipertrofia benigna de la próstata, pero no hay evidencia concluyente de que las tasas de cáncer de próstata aumenten en esta condición clínica. Lo mismo ocurre con el cáncer de mama(4).

- Gigantismo: Es una afección rara que ocurre en el $5 \%$ de los casos de tumores de la glándula pituitaria (somatotropinoma). Típicamente, se manifiesta en la segunda década de vida, las características clínicas aparecen cuando la hipersecreción de GH ocurre antes del cierre de los cartílagos epifisarios y resulta en un crecimiento lineal excesivo en los niños, altura con más de tres desviaciones estándar por encima de la media para la edad o más de dos desviaciones estándar de la altura ajustada a los padres (2). Las mutaciones del gen AIP se encuentran en el 29-50 \% de los casos de gigantismo. Recientemente se describió un nuevo síndrome llamado acrogigantismo ligado a X (XLAG), este caso de gigantismo infantil tiene un fenotipo clínico grave, con un aumento de la velocidad de crecimiento que inicia a los 2-3 meses de edad (2).

\section{Hallazgos radiológicos craneofaciales e intracraneales}

\subsection{Adenomas hipofisarios e interrupción del tallo hipofisario}

En más del $95 \%$ de los pacientes, la acromegalia se deriva de un adenoma hipofisario funcional (9), causante de un exceso en la producción de GH (hormona del crecimiento) el cual, en caso de no ser tratado, se asocia a una mayor mortalidad. Los macroadenomas ( $>10$ $\mathrm{mm}$ ) aparecen hasta en el $61 \%$ de los casos (figura 1), en el $39 \%$ restante se han observado microadenomas (10), así como un aumento del tamaño de la silla turca secundario a la expansión tumoral (6).

Se han informado casos de pacientes acromegálicos con hiperprolactinemia. Se debe tener en cuenta que la prolactina posee una estructura de aminoácidos primaria similar a la de la hormona del crecimiento $(\mathrm{GH})$, por lo cual compite débilmente por los receptores de unión a la $\mathrm{GH}$, además de tener propiedades biológicas similares a las de la hormona del crecimiento. Debido a su acción promotora del crecimiento en diversos tejidos y estructuras, la hiperprolactinemia en pacientes acromegálicos se debe a la secreción de la prolactina o $\mathrm{GH}$ 
por parte del tumor, la cual se observa hasta en el $50 \%$ de los casos o a la hiperprolactinemia secundaria a la compresión e interrupción del tallo hipofisario por el adenoma $(11,12)$ (figura 2).

\subsection{Aumento del espesor de la bóveda craneana frontal y occipital}

Para medir en tomografía computarizada (TC) el espesor de la bóveda craneana, se toma al nivel más alto de las alas mayores del esfenoides, se traza una línea transversa que conecta estos dos reparos anatómicos y una línea perpendicular a esta, posteriormente se traza una bisectriz desde la intersección de estas líneas hacia el hueso frontal y en este punto se realiza la medición del espesor de la bóveda craneal a nivel frontal. Para medir el espesor occipital se traza una línea transversa a nivel de la proyección ósea más anterior del hueso occipital y cuando esta línea está en contacto con la cortical ósea externa se traza un ángulo recto desde este punto equidistante y esta es la medida de la bóveda craneal a nivel occipital (figura 3) (9).

Aparte de las alteraciones mencionadas, se ha informado en la literatura una asociación entre acromegalia y la malformación Chiari, la cual propone que el sobrecrecimiento óseo reduce el volumen de la fosa posterior, pero estos datos deben ser confirmados con estudios adicionales (9).

Estas medidas demuestran que los pacientes con acromegalia tienen un engrosamiento de la bóveda craneana frontal de 1,12 +/- 0,43 $\mathrm{cm}$ y de la cortical óseo-occipital de $0,75+/-0,28 \mathrm{~cm}$. Estos datos difieren de los valores obtenidos para la población sin acromegalia, en quienes los valores del espesor de la bóveda craneal frontal son de $0,67+/-0,27 \mathrm{~cm}$ y de $0,55+/-0,14 \mathrm{~cm}$ para el espesor occipital (9) (tabla 1).

Tabla 1. Medidas de la cortical ósea frontal y occipital en pacientes con y sin acromegalia

\begin{tabular}{|c|c|c|}
\hline Cortical ósea & Valores normales & $\begin{array}{c}\text { Valores en } \\
\text { acromegalia }\end{array}$ \\
\hline Frontal & $0,67+/-0,27 \mathrm{~cm}$ & $1,12+/-0,43 \mathrm{~cm}$ \\
\hline Occipital & $0,55+/-0,14 \mathrm{~cm}$ & $0,75+/-0,28 \mathrm{~cm}$ \\
\hline
\end{tabular}

\subsection{Hiperneumatización de los senos paranasales}

La medición anteroposterior del seno esfenoidal en los pacientes con acromegalia es de 3,31+/- 0,62 cm, mientras que en los pacientes sin la patología la medida es de 2,92+/- 0,71 cm. Para el seno maxilar, las medidas en pacientes con acromegalia fueron de 4,22+/- 0,30 cm en comparación con los pacientes sin la patología cuyo valor es de 4,06 +/- 0,24 cm (9) (tabla 2) (figuras 4 y 5).

El aumento del diámetro del seno esfenoidal es una variante de gran importancia al momento de realizar la intervención transesfenoidal, porque dificulta el abordaje quirúrgico en la resección de los adenomas (9).

\section{Tabla 2. Diámetro anteroposterior (AP) del seno esfenoidal y maxilar en pacientes con y sin acromegalia}

\begin{tabular}{|c|c|c|}
\hline Seno paranasal & $\begin{array}{c}\text { Valores } \\
\text { normales }\end{array}$ & $\begin{array}{c}\text { Valores en } \\
\text { acromegalia }\end{array}$ \\
\hline Esfenoidal & $2,92+/-0,71 \mathrm{~cm}$ & $3,31+/-0,62 \mathrm{~cm}$ \\
\hline Maxilar & $4,06+/-0,24 \mathrm{~cm}$ & $4,22+/-0,30 \mathrm{~cm}$ \\
\hline
\end{tabular}

\subsection{Alteraciones faciales}

En los pacientes con acromegalia se observan diversas alteraciones en la estructura ósea facial, como el prognatismo, patrones de maloclusión, engrosamiento de los labios, ensanchamiento del maxilar, prominencia superciliar, macroglosia (causante de apnea obstructiva del sueño), diastemas dentales e hipertrofia del hueso nasal; de igual manera, se han informado diversos casos en los que, adicionalmente, se presenta hipertrofia mucosa, engrosamiento de la voz por la hipertrofia laríngea y pólipos de los senos paranasales (figura 6) $(6,9)$.

La mayoría de los pacientes tienen un cuadro clínico de 5 a 10 años de evolución con cambios en la arquitectura facial antes de que se les diagnostique la acromegalia. Por lo cual, ante un prognatismo mandibular bilateral simétrico - la rama mandibular generalmente se encuentra más afectada que el cuerpo mandibular-, en los estudios por TAC o RM se debe tener en cuenta la acromegalia como diagnóstico diferencial (figura 7) (6).

\subsection{Afectación orbitaria}

La afectación orbitaria en la acromegalia es un hallazgo poco frecuente, hasta el 5,7 \% de los pacientes pueden tener defectos campimétricos, proptosis o diplopía debido a la miopatía restrictiva $\mathrm{o}$, menos frecuentemente, secundarios a parálisis de nervios craneales (13).

Los hallazgos por imágenes son un agrandamiento difuso y simétrico de los músculos extraoculares, el grado de hipertrofia está relacionado con la duración de la enfermedad y no con los niveles hormonales (13). Los estudios histopatológicos muestran una hipertrofia de las fibras tipo 1 y atrofia de las fibras tipo 2, como resultado directo del exceso de secreción de hormona del crecimiento (13) (figura 8).

\section{Otras técnicas de imagen}

Aunque la RM con medio de contraste paramagnético es la técnica de elección en la caracterización de los adenomas hipofisarios (identifica adenomas de hasta $2 \mathrm{~mm}$ de diámetro), la tomografía por emisión de positrones (PET) ofrece una imagen anatómica y funcional del adenoma, ya que los ligandos usados en la PET aportan información para la diferenciación de tejido tumoral adenomatoso de otras lesiones, como quistes, fibrosis, necrosis, sangrado y otros tumores, como el craneofaringioma (14).

En los pacientes con acromegalia, la PET muestra un alto índice metabólico tumoral que disminuye de manera considerable al instaurar tratamiento médico con análogos de somatostatina, por lo que se ha propuesto como herramienta para monitorizar la eficacia del tratamiento(14).

La gammagrafía corporal total es una modalidad de imagen útil en la detección de patología hipofisaria, adicionalmente permite inferir 
patologías asociadas a la acromegalia, como la hiperplasia condílea secundaria a los efectos del IGF-1 sobre la articulación temporomandibular $(15,16)$ (figura 9).

\section{Discusión y conclusiones}

La acromegalia es una patología causada por hipersecreción crónica de la hormona del crecimiento $(\mathrm{GH})$ y del factor de crecimiento similar a la insulina 1 (IGF-1), caracterizada por un compromiso multisistémico, en especial en los sistemas cardiovascular, respiratorio y metabólico, con las subsecuentes complicaciones ya mencionadas.

Adicionalmente, en estos pacientes se encuentran diversos tipos de malformaciones craneofaciales, las cuales conllevan un mayor riesgo de complicaciones quirúrgicas al momento de realizar la resección de los adenomas hipofisarios por vía transesfenoidal, razón por la cual, el radiólogo juega un papel fundamental en la identificación de las características craneofaciales mediante los estudios por TAC y RM, así como, en la detección de adenomas hipofisarios, puesto que su detección les brinda a los pacientes con acromegalia la oportunidad de un diagnóstico precoz de la enfermedad, y un tratamiento oportuno, que prevenga las complicaciones de la enfermedad.

\section{Reconocimientos}

Agradecimientos especiales a la doctora Luz Kelly Anzola Fuentes, especialista en Medicina nuclear, jefe del Servicio de Medicina nuclear de la Clínica Reina Sofía y Clínicas Colsanitas.

\section{Referencias}

1. De Herder WW. The history of acromegaly. Neuroendocrinology. 2016;103:7-17.

2. Vilar L, Vilar CF, Lyra R, Lyra R, Naves LA. Acromegaly: Clinical features at diagnosis. Pituitary. 2017;20(1):22-32.

3. Chandna A, Islam N, Jabbar A, et al. Clinical features and outcome of surgery in 30 patients with acromegaly. J Pak Med Asoc. 2004;54(6):315-9.

4. Scacchi M, Cavagnini F. Acromegaly. Pituitary. 2006;9(4):297-303.

5. Karakıs D, Yılmaz B, Dogan A, Yetkin I, Bek B. The bite force and craniofacial morphology in patients with acromegaly : A pilot study. Med Oral Patol Oral Cir Bucal. 2014;19(1):e1-7.

6. Gosau M, Vogel C, Moralis A, Proff P, Kleinheinz J, Driemel O. Mandibular prognathism caused by acromegaly-a surgical orthodontic case. Head \& Face Medicine. 2009;5:16

7. Lavrentaki A, Paluzzi A, Wass JAH, Karavitaki N. Epidemiology of acromegaly: review of population studies. Pituitary. 2017; 20(1):4-9.

8. Buchfelder M, Schlaffer SM. The surgical treatment of acromegaly. Pituitary. 2017;20(1):76-83.

9. Ebner FH, Kürschner V, Dietz K, Bültmann E, Nägele T, Honegger J. Craniometric changes in patients with acromegaly from a surgical perspective. Neurosurg Focus. 2010;29(4):1-5.

10. Zada G, Cavallo L, et al. Transsphenoidal surgery in patients with acromegaly: Operative strategies for overcoming technically challenging anatomical variations. Neurosurg Focus. 2010;29:18-20.

11. Valenta LJ, Elias AN. Clinical acromegaly with undetectable growth hormone and hyperprolactinemia. J Natl Med Assoc. 1987;79(5):555-60.

12. Katznelson L, Atkinson JL, Cook DM, Ezzat SZ, Hamrahian AH, Miller KK, American Association of Clinical Endocrinologists. American Association of Clinical Endocrinologists medical guidelines for clinical practice for the diagnosis and treatment of acromegaly - 2011 update. Endocr Pract. 2011;17(Suppl 4):1-44.

13. Heireman S, Delaey C, Claerhout I, Decock CE. Restrictive extraocular myopathy: A presenting feature of acromegaly. Indian J Ophthalmol. 2011;59:517-9.

14. García JCF, Juan CS, Ballester AH. Aportación de la tomografía por emisión de positrones al diagnóstico de un caso de acromegalia. Endocrinol Nutr. 2008;55(4):175-7.

15. Lugo G, Pena L, Cordido F. Clinical manifestations and diagnosis of acromegaly. Int J Endocrinol. 2012;2012:540398.

16. Hodder SC, Rees JI, Oliver TB, Facey PE, Sugar AW. SPECT bone scintigraphy in the diagnosis and management of mandibular condylar hyperplasia. Br J Oral Maxillofac Surg. 2000;38:87-93.

\section{Correspondencia}

Luis Manuel Alejandro Acosta Rosas

Carrera 66 \# 23-46

Clínica Universitaria Colombia

Departamento de Radiología e Imágenes Diagnósticas

Bogotá, Colombia

acostarosas@gmail.com

Recibido para evaluación: 9 de abril de 2019

Aceptado para pubzlicación: 20 de septiembre de 2019 